\title{
FROM SPECIFICATION TO MEASUREMENT: THE BOTTLENECK IN ANALOG INDUSTRIAL TESTING
}

\section{R.J. van Rijsinge, A.A.R.M. Haggenburg, C. de Vries}

Philips Components

Business Unit Consumer IC

Gerstweg 2, 6534 AE Nijmegen

The Netherlands

\author{
H. Wallinga
}

\author{
University of Twente \\ Fac. of Electrical Engineering \\ Postbox 217, 7500 AE Enschede \\ The Netherlands
}

\begin{abstract}
The translation of the specification of an analog device into the necessary set of measurements to be carried out by an industrial test facility, is discussed. Algorithms are developed to compute the number of testvectors needed to guarantee a certain parameter and to compare several possible testmethods, based on accuracy. An important input for these algorithms: the measurement error is also discussed.
\end{abstract}

\section{Introduction}

An important part of the development of an Integrated Circuit (IC) for mass-production, is the development of an Industrial Test Facility (ITF). The task of the ITF is to guarantee the correct functioning of a product. Bad products must be rejected by the ITF. For analog circuits, in order to fulfil this task, the product is compared with the specification of the IC. Therefore the development of the ITF is based on the specification of the IC. The translation of a specification into the necessary set of measurements, to be carried out by the ITF, is a very dedicated task. This is the main subject of this paper.

If we take a look at several specifications of analog circuits, we distinguish two kinds of specifications:

1. Functional Specification: this part describes the main function of the $I C$. Testing this part is done very straightforward. An example is the test if an oscillator oscillates, regardless of the frequency.

2. Parameter Specification: this part describes the ratings for several parameters of the IC. Opposite to the functional testing, for parameter testing the accuracy of the test is of high importance.
Because the main testing problems arise for parameter specifications, the functional specification will not be further discussed. The accuracy of the parameter specification is defined by the measurement error. Therefore a trade-off between test methods can be made based on accuracy.

The parameter specification can again be split into two classes:

1. Single Parameter Specification: for such a parameter, all the conditions for the measurements, like input voltage and -frequency, temperature, etc. have a predefined value. An example is the specification of the DC-gain of an amplifier at $25^{\circ} \mathrm{C}$, and $10 \mathrm{mV}$ input voltage.

2. Interval Parameter Specification: here, at least one of the conditions is varying: for example, the specification of the maximum DC-gain deviation of an amplifier for a range of inputvoltages at $25^{\circ} \mathrm{C}$ (linearity).

Because the ITF can only execute single parameter measurements, the interval parameter specification must be translated into several single parameter specifications. This is discussed in section 2. Section 3 describes a method to compare several testmethods based on accuracy. The basis to determine the accuracy, the measurement error will be looked at in section 4 . The final conclusions are given in section 5 .

\section{From interval to parameter specification}

Consider the measurement of a linearity factor. In this case, there is a relationship like:

$$
y=\beta^{\star} x+a
$$

Here, $x$ is the parameter which can be applied, 
$a$ is an arbitrary constant, $y$ is the parameter which can be measured and $\beta$ is the parameter which has to be fixed. For several $x$ values it is possible to measure the value of $y$ and compute a value for $\beta$. This is illustrated by figure 1 .

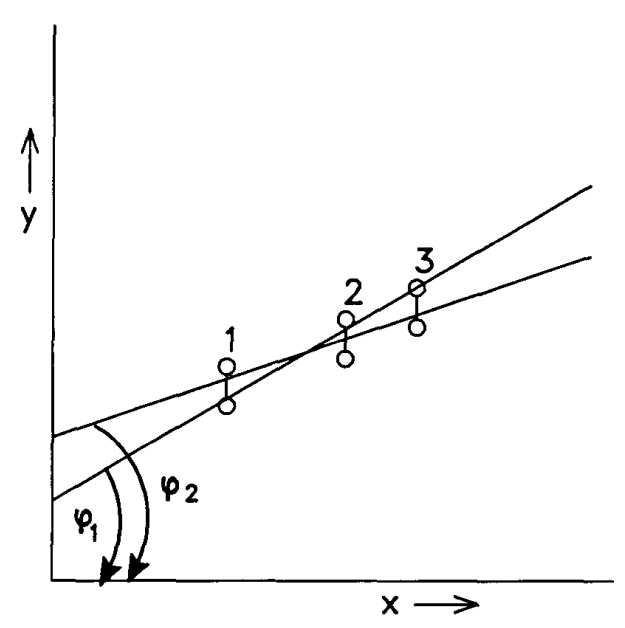

Figure 1

In this case three points (1 to 3) are taken from a linear curve. Due to the measurement error, the real value for $y$ will lie somewhere between the two dots. The error in the supplied variable $x_{i}$ is neglected compared to the error in $y_{i}$. The angle of the curve, which is a measure for $\beta$, can vary between $\phi_{1}$ and $\phi_{2}$. If the number of points, or testvectors, is increased, the uncertainty in the predicted curve, which, in this case determines the linearity error $(\Delta \beta)$, is decreased. Because a non-linear relation can easely be transformed into a linear one, the restriction to a linear relationship is not a fundamental one.

For every set of values $x_{i}$ and measured values $y_{i}$, one gets an estimate of $\beta$ (written down as b). The real value of $\beta$ will be somewhere near this estimated value. It is possible to derive a confidence interval for the value of $\beta$, i.e. this interval will, with a certain confidence, for instance $90 \%$ certainty, contain the real value of $\beta$. This interval can be computed, using the estimate of $\beta$ belonging to some set of testvectors $\left(x_{i}, y_{i}\right)$. This is described by:

$$
y_{i}=\beta\left(x_{i}-\bar{x}\right)+\varepsilon_{i}+\mu_{y}
$$

with $\varepsilon_{i}$ the measurement error and $\mu_{y}$ the mean value of $y$.

It is quite logical that an increase in the number of testvectors, results in a decrease of the width of the confidence interval. Another influence on the width of the interval is the measurement error. If the measurement error is small, the width of the confidence interval will be small. If we take both influences into account, the confidence interval is given by the equation [1]:

$b-\beta=t(n-2 ; \alpha / 2) \cdot \sqrt{ } \operatorname{var}(b)$

(3),

with $\mathrm{t}$ : students-t distribution with $\mathrm{n}-2$ degrees of freedom and $\alpha$ the $100(1-\alpha) \%$ confidence interval for the error in $b$.

The variance of $b$ is calculated from the estimate for $\beta$ :

$$
\begin{aligned}
& b=\frac{\sum_{i=1}^{n}(X i-\bar{X})(Y i-\bar{Y})}{\sum_{i=1}^{n}(X i-\bar{X})^{2}} \\
& \beta+\frac{\sum_{i=1}^{n}(x i-\bar{x}) \varepsilon_{i}}{\sum_{i=1}^{n}(X i-\bar{X})^{2}}
\end{aligned}
$$$$
b=\underline{i=1}=
$$

So, the estimate $b$ is equal to the value of $\beta$ plus an error component. We assume all errors to be equal normal distributed as $\mathrm{N}\left(0, \sigma_{\mathrm{c}}{ }^{2}+\sigma_{\mathrm{u}}{ }^{2}\right)$, with $\sigma_{c}{ }^{2}$ the variance part which is fully correlated to the other errors, and $\sigma_{u}{ }^{2}$ the variance part which is totally uncorrelated. Because we only look at the difference between two measurements, the systematic error is eliminated and the variance of $b$ is:

$$
\begin{aligned}
& \operatorname{var}(\mathrm{b})=\operatorname{var}\left(\frac{\sum_{i=1}^{n}(\mathrm{xi}-\overline{\mathrm{X}}) \varepsilon_{\mathrm{i}}}{\sum_{\mathrm{i}=1}^{n}(\mathrm{Xi}-\overline{\mathrm{X}})^{2}}\right)= \\
& \frac{\sigma_{\mu}{ }^{2}}{\sum_{\mathrm{i}=1}^{n}(\mathrm{Xi}-\overline{\mathrm{X}})^{2}}
\end{aligned}
$$


If we take $n$ equal spaced values of $x$ in the interval $\left(x_{m, n}, x_{m a x}\right)$ and define $R=\left|x_{m i n}-x_{m a x}\right|$ so $\left|x_{i}-x_{i-1}\right|=R /(n-1)$ then:

$$
\begin{aligned}
& \sum_{i=1}^{n}(X i-\bar{X})^{2}= \\
& 2^{*}\left(\frac{R}{n-1}\right)^{2} * \sum_{i=1}^{L}\left(\frac{n+1}{2}-i\right)^{2}
\end{aligned}
$$

$$
\text { with } L=n / 2 \text { for } n=\text { even; }
$$

$$
=(n-1) / 2 \text { for } n=\text { odd. }
$$

The measurement error $\sigma_{u}$ is in general proportional to the range which has to be measured, and defined as a percentage ( $\delta$ ) of this maximum. Because the range of $y$ is equal to $b^{\star} R$, this can be expressed as:

$$
\sigma_{\mathrm{u}}^{2}=(\delta \mathrm{bR})^{2}
$$

If we combine $3,5,6$ and 7 we'll get:

$$
\begin{aligned}
& \Delta b / b=t(n-2 ; a / 2) \cdot \delta K \\
& \text { with } \\
& K=\frac{n-1}{\sqrt{ } 2}\left\{\sum_{i=1}^{L}\left(\frac{n+1}{2}-i\right)^{2}\right\}^{-1 / 2}
\end{aligned}
$$

TABLE 1 Number of measurements versus the variance of the measurement error.

\begin{tabular}{|c|c|c|c|}
\hline $\mathrm{n}$ & $\mathrm{K}$ & $\mathrm{t}(\mathrm{n}-2 ; 0.005)$ & $\delta$ \\
\hline 3 & 1.41 & 63.657 & $0.01 \%$ \\
4 & 1.34 & 9.925 & $0.08 \%$ \\
5 & 1.26 & 5.841 & $0.14 \%$ \\
6 & 1.20 & 4.604 & $0.18 \%$ \\
7 & 1.13 & 4.032 & $0.22 \%$ \\
8 & 1.08 & 3.707 & $0.25 \%$ \\
9 & 1.03 & 3.499 & $0.28 \%$ \\
10 & 0.99 & 3.355 & $0.30 \%$ \\
\hline
\end{tabular}

The relative error in the estimate of $\beta$ depends only on: the relative error of the measurement $(\delta)$ and the number of measurement points (n). An increase in the number of measurements $n$ will result in a decrease of $K$. For an infinite number of measurements $\mathrm{K}$ will become zero. In table 1 some values of $\delta$ with the corresponding values of $n$ are given for a $1 \%$ relative error in $\beta$, with $99 \%$ confidence.

If the relative measurement error is small, only three testvectors are needed to compute the value of $\beta$ with $1 \%$ accuracy. If the measurement error is relative large, more testvectors are needed.

\section{Conclusion:}

Regardless of the nature of $x$ and $y$, it is possible to make a trade-off between the accuracy of the measurement, the number of test vectors $(x, y)$ and the relative error in the result $(\Delta b / b)$.

\section{Comparison of test methods}

After the translation of the interval parameters, a testspecification of strictly single parameter specifications remains. Now, the specific testmethods for every parameter have to be defined. Because there are many ways to test a specific parameter, a selection between the testmethods must be made. In this paragraph, a selection method based on accuracy is proposed. The model, used to represent the relation between the measurement and the specified parameter is based on the model discussed in [2]. The modification is made for two reasons: first of all, with the new model it is possible to have multiple measurements for the prediction of one parameter. This is the case for interval specifications, but also for relative parameters like gain. To measure the gain of an amplifier, two measurements are needed to define one parameter (one for the reference level and one for the output level). Secondly, correlation between measurement errors is taken into account. The proposed model is:

$$
\begin{aligned}
& \Delta \mathrm{p}_{i}=\mathrm{S}_{i j}\left(\Delta \mathrm{x}_{j}+\mathrm{e}_{j}\right) \\
& \text { with: } \mathrm{S}_{i j}=\partial \mathrm{p}_{\mathrm{i}} / \partial \mathrm{x}_{j} \\
& \text { and: } \\
& \Delta \mathrm{p}_{i}: \text { deviation in the specified parameter. } \\
& \Delta \mathrm{x}_{j}: \text { deviation in the measured parameter. } \\
& \mathrm{e}_{j}: \text { measurement error in the parameter } \\
& \mathrm{x}_{j} . \\
& \mathrm{S}_{i j}: \text { relation between the specified } \\
& \text { parametervalue } \mathrm{p}_{i} \text { and the measured } \\
& \text { parametervalue } \mathrm{x}_{j}
\end{aligned}
$$

To select the best testmethods, the deviation of the measured variable $\Delta x$ is put to zero. In matrix notation we'll get: 


$$
\Delta \mathbf{p}=\mathbf{S}^{*} \mathbf{e}
$$

The variance of the inaccuracy $\Delta p$ is equal to:

I $\left[\sigma_{p}{ }^{2}\right]=\mathbf{S}^{\star} \mathbf{E}_{v, c} \mathbf{S}^{\top}$

with:

$I:|\times|$ identitymatrix; $\mid$ is equal to the number of specified parameters.

$\mathbf{S}$ : the matrix of the elements $S_{i j}$.

$\mathbf{S}^{\top}$ : the transpose of the matrix $\mathbf{S}$.

$\mathbf{E}_{\mathrm{v}, \mathrm{c}}$ : the variance-covariance matrix of the measurement errors $e_{j}$.

The main target is to minimize the total variance given by:

$$
\sum_{i=1}^{I} \sigma_{p_{i}}{ }^{2}
$$

To compare different testmethods (11) can be computed for every possible combination of the measured parameters. Because this is computational inefficient, a selectioncriterion is derived. Let (13) represent formula (11) for the measured parameters $x_{1} \ldots x_{k}, x_{k+1} \ldots x_{j}$ and (14) for the measured parameters $x_{1}^{\prime} \ldots x_{L}^{\prime}, x_{k+1} \ldots x_{J}$ with $L \leq K$ :

$$
\begin{aligned}
& I\left[\sigma_{p}{ }^{2}\right]=\left[\begin{array}{lll}
d x & d c
\end{array}\right]\left[\begin{array}{ll}
P & Q
\end{array}\right]\left[(d x)^{\top}\right] \\
& {\left[\begin{array}{ll}
Q^{\top} & R
\end{array}\right]\left[\begin{array}{l}
(d c)^{\top} \\
]
\end{array}\right.}
\end{aligned}
$$

$$
\begin{aligned}
& \text { I[ } \left.\sigma_{p}{ }^{2}\right]=\left[\begin{array}{llll}
\mathbf{d x} & \mathbf{d c}] & {\left[\begin{array}{ll}
\mathbf{S} & \mathbf{T}
\end{array}\right]\left[\left(\mathbf{d x}^{\prime}\right)^{\top}\right]}
\end{array}\right. \\
& {\left[\begin{array}{ll}
\mathbf{T}^{\top} & \mathbf{R}
\end{array}\right]\left[(\mathrm{dc})^{\top}\right]}
\end{aligned}
$$

with:

$\mathbf{d x}$ : the matrix of the elements $\partial \mathrm{p}_{\mathrm{i}} / \partial \mathrm{x}_{\mathrm{j}}$ with $1 \leq \mathrm{j} \leq \mathrm{K}$;

dc : the matrix of the elements $\partial \mathrm{p}_{\mathrm{i}} / \partial \mathrm{x}_{\mathrm{j}}$ with $\mathrm{K}+1 \leq \mathrm{j} \leq \mathrm{J}$;

$\mathbf{d x}^{\prime}$ : the matrix of the elements $\partial p_{i} / \partial x_{j}{ }_{j}$ with $1 \leq j \leq L$

$P$ : the variance - covariance submatrix of $e_{j}$ with $1 \leq j \leq K$;

$Q$ : the covariance submatrix of $\left(e_{i}, e_{j}\right)$ with $1 \leq j \leq K$ and $K+1 \leq i \leq J$;

$\mathbf{R}$ : the variance - covariance submatrix of $e_{j}$ with $\mathrm{K}+1 \leq \mathrm{j} \leq \mathrm{J}$;

$S$ : the variance - covariance submatrix of $e^{\prime}$, with $1 \leq j \leq L$;

$T$ : the covariance submatrix of $\left(e_{1}, e^{\prime}{ }_{j}\right)$ with $1 \leq j \leq L$ and $K+1 \leq i \leq J$;

The criterion for minimal variance is given by:

$$
\sum_{i=1}^{I} \sigma_{p_{i}}{ }^{2}\left|-\sum_{i=1}^{I} \sigma_{p_{i}}{ }^{2}\right|=\mathbf{D}
$$

Using some standard matrix operations, the following equation is derived from (13), (14) and (15):

$$
\begin{aligned}
& \sum_{d} \mathbf{D}=\sum_{d}[\mathbf{d x}] \mathbf{P}[\mathbf{d x}]^{\top}+2^{*} \sum_{d}[\mathbf{d x}] \mathbf{Q}[\mathbf{d c}]^{\top}- \\
& \left\{\sum\left[\mathbf{d} \mathbf{x}^{\prime}\right] \mathbf{S}\left[\mathbf{d} \mathbf{x}^{\prime}\right]^{\top}+2_{d}^{*} \sum\left[\mathbf{d} \mathbf{x}^{\prime}\right] \mathbf{T}[\mathbf{d} \mathbf{c}]^{\top}\right\}
\end{aligned}
$$

with $\sum$ the summation of the diagonal d elements of the resulting matrix.

The first sum contains the contribution of the first $\mathrm{k}$ parameter measurement errors to the variance of the errors $\sigma_{\mathrm{p}}{ }^{2}$, analog to (11). The second sum gives the contribution of the interaction between these errors and the measurement errors of the parameters equal for both sets. The term between brackets gives this contribution but now for the alternative parameter set with the parameters $X_{1}^{\prime}{ }_{1} \ldots \mathbf{X}_{\mathrm{L}}^{\prime}$. The selectioncriterion is:

$$
\sum_{d} \mathbf{D}<?>0
$$

and a conclusion whether it is advantageous to measure $x_{1} \ldots x_{k}$ instead of $x_{1}^{\prime} \ldots x_{L}^{\prime}$ can be drawn.

The efficiency of this algorithm depends on the ratio between the total number of measured parameters $\mathrm{J}$ and the number of parameters which are different in both sets: $K$. The reduction in the total number of multiplications needed to compute $\mathbf{D}$ compared to the computation of (11) directly, with $\mathrm{L}=\mathrm{K}$ is equal to:

$$
\text { Reduction }=\frac{-\mathrm{J}+1}{\mathrm{~K}+1}
$$

This algorithm is meant to be used in a CAD environment. For a reliable output, the variance - covariance matrix of the measurement error is needed. This aspect will be discussed in the next section.

\section{The measurement error}

To fill in the variance - covariance matrix of the measurement errors, knowledge of the systematic and stochastic error components of 
a measurement is needed. The relation between the variance and covariance elements and these error components are:

$$
\begin{aligned}
& \operatorname{var}\left(e_{i}\right)=\sigma_{s t o c h}{ }^{2}+\sigma_{s y s t}{ }^{2} \\
& \operatorname{cov}\left(e_{i}, e_{j}\right)=\sigma_{s y s t}{ }^{2}
\end{aligned}
$$

Because the total testsystem is very complex, the limits for the error components have to be set by experimental data. If we test several products on several identical testsystems a prediction for the variances and covariances of the error components can be made. The main question involves the relation between the reliability of the estimate of the variance in relation to the complexity of the experiment. There are two separate cases which will be discussed in the next sections.

\subsection{Equal error behavior for all testsystems and products}

First of all, we assume no significant difference between several testsystems, and no influence of the product on the error behavior of the measurement. With this assumption, it is possible to derive a $100(1-\alpha) \%$ confidence interval for the variance of the measurement error by testing one product on one testsystem several times. If a relative measurement is made, the systematic error component is eliminated, and a prediction of $\sigma_{\mathrm{stoch}}{ }^{2}$ can be made. For an absolute measurement, the variance consists of both stochastic and systematic errors. If we measure the product $N$ times, the sample variance can be calculated as [1]:

$$
S_{x}{ }^{2}=\left\{\sum_{i=1}^{n}(X i-\bar{X})^{2}\right\} /(N-1)
$$

and the confidence limits for the variance are [1]

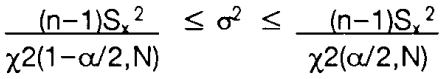

with $\chi^{2}(\mathrm{a}, \mathrm{N})$ the $\chi^{2}$-distribution with $\mathrm{n}$ degrees of freedom.

Unfortunately, in most practical cases the assumption of no influence from testsystem and product on the measurement error is violated. In this case the second method must be used to derive a confidence interval for the variance of the measurement error.

\subsection{Nonequal error behavior between testsystems and products}

If there is a significant influence of the product and the choice of the testsystem on the error behavior, the following model for the measurement of one parameter can be used:

$$
x_{i j}=\mu+a_{i}+e_{i j}
$$

with:

$\mu$ : mean value of the parameter $x$.

$a_{i}$ : influence of testsystem $i ; i \in[1 . .1]$

$e_{i j}$ : influence of product $j$ on testsystem $\mathrm{i} ; \mathrm{j} \in\left[1 . . \mathrm{n}_{\mathrm{i}}\right]$

Because $a_{i}$ is independent of $e_{i j}$, the variance of the parameter $x$ is

$$
\sigma_{x}^{2}=\sigma_{a}^{2}+\sigma_{e}^{2}
$$

Here, $\sigma_{a}{ }^{2}$ represents the variance due to the testsystem and $\sigma_{\mathrm{e}}{ }^{2}$ the variance due to the productionspread of the process. In [3] a confidence interval for the ratio $b=\sigma_{a}^{2} / \sigma_{e}{ }^{2}$ for this model is derived:

$$
\frac{n-1}{\mathbf{l}-1} * \frac{\sum_{i=1}^{I} g_{i}\left(\bar{x}_{1}-\mathbf{x}\right)^{2}}{\sum_{i=1}^{I} \sum_{j=1}^{n_{i}}\left(X_{i j}-\bar{X}\right)^{2}}=f_{p}
$$

with:

$$
\begin{aligned}
& \mathrm{g}_{i}=\mathrm{n}_{i} /(\mathrm{bn}+1) \\
& \bar{x}_{i}=\sum_{j=1}^{n_{i}} X_{i j} / n_{i} \\
& \mathbf{x}=\sum_{i=1}^{I} \frac{g_{i} \bar{x}_{i}}{\sum_{i=1}^{l} g_{i}} \\
& \mathbf{n}=\sum_{i=1}^{I} n_{i}
\end{aligned}
$$

The solution of (24) for $f_{\mathrm{p}}=f_{1}$ gives the lower bound on $b$. The upper bound is given by the solution of (24) for $f_{p}=f_{h}$. The values of $f$ are found using the F-test table with $\mathbf{I - 1}$ and $n-1$ degrees of freedom and a confidence level $\alpha$ : $\mathrm{F}\left(f_{1}, \mathrm{I}-1, \mathbf{n}-\mathrm{I}\right)=\alpha / 2, F\left(f_{\mathrm{h}}, \mathrm{I}-1, \mathbf{n}-\mathrm{I}\right)=1-\alpha / 2$. If $n_{i}=n_{j}=a$ equation (24) can be simplified: 


$$
\begin{aligned}
& \frac{n-1}{I-1} \frac{\sum_{i=1}^{I} g_{i}\left(\bar{x}_{i}-x\right)^{2}}{\sum_{i=1}^{I} \sum_{j=1}^{n_{i}}\left(X_{i j}-\bar{X}\right)^{2}}= \\
& \begin{array}{l}
\frac{a}{b^{*} a+1} * \frac{n-1}{I-1} * \frac{\sum_{i=1}^{I}\left(\bar{x}_{i}-x\right)^{2}}{\sum_{i=1}^{I} \sum_{j=1}^{a}\left(X_{i j}-\bar{x}\right)^{2}} \\
\frac{a}{b^{*} a+1} * \frac{M S a}{M S e}=f_{p}
\end{array}
\end{aligned}
$$

with: MSa: Mean Square of a and MSe: Mean Square of e,

$F\left(f_{l}, I-1,(a-1) I\right)=\alpha / 2$,

$F\left(f_{h}, I-1,(a-1) l\right)=1-\alpha / 2$.

The width of the confidence interval for $b$ can be calculated:

$$
\left(b_{\max }-b_{\min }\right)=\frac{M S a}{M S e} *\left(\frac{1}{f_{h}}-\frac{1}{f_{1}}\right)
$$

The ratio of mean squares is the estimate for $b$. In table 2 the value of $\left(1 / f_{1}-1 / f_{h}\right)$ is given versus the number of testsystems (I) and the number of products (a) for a $90 \%$ confidence level $(\alpha=0.10)$. As we can see, for a reliable variance prediction, at least 10 testsystems must be involved in the experiment. This may cause practical problems.

\begin{tabular}{|c|ccccc|}
\hline \multicolumn{1}{c}{} & $\mathrm{a}=2$ & $\mathrm{a}=3$ & $\mathrm{a}=4$ & $\mathrm{a}=10$ & $\mathrm{a}=15$ \\
\cline { 2 - 6 } $\mathrm{I}=3$ & 19 & 19 & 19 & 19 & 19 \\
$\mathrm{I}=4$ & 8.9 & 8.6 & 8.4 & 8.3 & 8.2 \\
$\mathrm{I}=5$ & 6.1 & 5.7 & 5.5 & 5.3 & 5.3 \\
$\mathrm{I}=6$ & 4.7 & 4.4 & 4.2 & 4.0 & 4.0 \\
$\mathrm{I}=8$ & 3.4 & 3.1 & 3.0 & 2.8 & 2.8 \\
$\mathrm{I}=10$ & 2.8 & 2.5 & 2.4 & 2.3 & 2.2 \\
$\mathrm{I}=15$ & 2.1 & 1.8 & 1.7 & 1.6 & 1.6 \\
\hline
\end{tabular}

TABLE 2 The width of the confidence interval

\section{Conclusions}

To summarize this work, some final conclusions are given:

1. The specification of a circuit can be transformed into single parameter measurements, to be carried out by an industrial test facility. There is a trade-off between the number of measurements and the accuracy of the specified parameter. 2. A computational efficient trade-off between testmethods based on maximum accuracy can be made.

3. One of the aspects needed to make these tradeoffs: the measurement error can be predicted using one of the proposed experiments. Here, again there is a trade-off between the complexity of the experiment and the accuracy of the error prediction.

4. To predict the variance of the measurement error, a rather complex experiment must be carried out in order to get an acceptable confidence interval for the estimated variance.

\section{References}

[1] C.P. Cox, "A handbook of introductory statistical methods", 1987, John Wiley \& Sons, Inc., ISBN 0-471-81971-9

[2] G.J. Hemink, B.W. Meijer, H.G. Kerkhoff, "Taste, a tool for analog system testability evaluation". Proceedings of the international test conference, 1988, pp 829-838.

[3] L.R. Verdooren, "Statistical interference on variance components", Agricultural university of Wageningen, 1988.

Paper 7.2 